\title{
Fault identification in power cables by using travelling wave-based approach
}

\author{
M.NagaJyothi ${ }^{a}$, N.Mounika ${ }^{b}$, B.Puneeth ${ }^{\mathrm{c}}$, and T.Haripriya ${ }^{\mathrm{d}}$

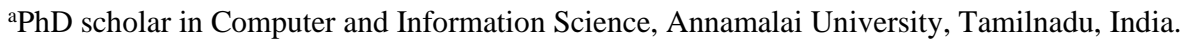 \\ ${ }^{\mathrm{b}}$ Assistant Professor in Computer Science Engineering, Annamalai University, Tamilnadu, India. \\ ${ }^{c}$ Assoicate Professor in Computer Science Engineering, Annamalai University, Tamilnadu, India. \\ Associate Professor in Manufacturing engineering, Annamalai University, Tamilnadu, India.
}

Article History: Received: 11 January 2021; Accepted: 27 February 2021; Published online: 5 April 2021

\begin{abstract}
Thefaults in the underground cables cause to generatetransients which propagate along the power cable as travelling waves. The velocity of the generated travelling wave and the time taken by a wave to reach the source point from the fault location enables us to calculate the fault distance value.In this paper a double endedtravelling wave based approach is used and a communication channel is designed to calculate the fault distance. A circuit-based model is developed in the EMTP-RV software to find out the fault distance from the source end for all types of faults. Further it is proved that the fault distance is unaffected by the change in ground resistance, various types of faults and the fault inception angle in the proposed method.

Keywords: Clarke's transformation, Double end method, Surge impedance, Travelling Wave, Undergroundcable,Wave velocity.
\end{abstract}

\section{Introduction}

The Power cables are widely used in many of the developed countries and in the urban areas as it creates an aesthetic and safe environment. The medium for power transmission has been changing from conventional mode of overhead lines to underground cables. The faults in the cable became more frequent due to the insulation aging, material faults and mechanical damage. The cost of the underground cable is about 5 to 10 times more compared to the overhead line for same rating and length of the line. Whenever a fault occurs in the underground cable it incurs huge loss to the system compared to overhead lines (Stringfield, 1957; Balamurugan, 2020; Sneha, 2020). So, it is always advisable to locate the fault immediately and clearit as soon as possible. But there arises a major problem while locating the fault in the underground cables, since the cable laying is hidden. Therefore, the study on the development of the techniques to locate faults in the power cables has been developed.

\subsection{Various methods to locate faults in cables}

Many techniques have been proposed to locate the faults in the overhead linesand in the underground cables (Latchoumi, 2020; Deepthi, 2020). Initially impedance based method is used where the voltages and currents are calculated and then sequence impedances are measured to findout the fault distance. This method is easy to implement but the drawback of this method isit doesn't provides the accurate results; the error is about 20tower span (Roostaee, 2017; V, 2020). For analysing the transient waveforms and to locate the fault, travelling wave based approach is proposed by many of the researchers as they are insensitive to the load flow, series compensation and transition resistance (Qin, 2007; Ezhilarasi, 2020). Travelling wave based approach is the widely used method in the overhead lines to locate the faults as it provides the accurate fault distance with an error of about one tower span. The travelling wave based method is further categorized in to two types namely, single ended fault locating technique and the double ended fault locating technique. Since many years single ended travelling wave method is used as it provides fast results and there is no need of any channelfor communication (Schweitzer, 2016). But the main drawback of this method is, it is hard to analyse the reflected waves produced from the fault end to the source. In turn, this makes it difficult to calculate the time taken by a wave to reach the fault point from the source (Liang, 2013; Schweitzer, 2016; Spoor, 2006). Then the double ended method is proposed which overcomes the drawback of single ended method and it gives the accurate results compared to the single ended method (Xiaoguang, 2011; Garikapati, 2020). Generally the fault location in cable is done in three steps. Initially the fault characteristics are determined by the insulation and the fault resistance values of the power cable, secondary the fault distance in the cable is identified by employing different techniques. Finally, route tracing and pin pointing is done to find the exact distance of the fault and the faulted cable is removed or rectified to make it to work under normal condition.

\subsection{Overview of the proposed work}

In this paper a double ended travelling wave based approach is implementedand a communication channel is designed in the EMTP-RV softwarewhich enables us to locate the fault in the underground cables. This method is implemented while the system is under energised condition and this is the main advantage of this method. Earlier in (Schweitzer , 2014) the fault location in overhead lines has been found out using the travelling wave based 
double ended type-D method.To the continuation of that work in this paper the double ended travelling wave based approach is used to locate fault in the underground cable(Xiaoguang, 2011). A Simulink model is developed to calculate the fault distance value in the EMTP-RV software. The faults in the underground cables causes to produce voltage and current transients in the system which travels along the cable called as travelling waves. The coupling capacitor voltage transformer (CCVT) and the conventional current transformer (CCT) are used generally for the voltage and current measurements. But the CCVTs have poor frequency response compared to CCTs. So, the current transient waveforms are taken here from the conventional CTs to locate the fault point(Schweitzer , 2014). To simplify the analysis,three phase current transient components are converted to two phase using the phase to modal transformations. The clarke's transformation enables us to convert three phase vector components to two phase orthogonal vectors. The high pass filter is used to eliminate the low frequency or power system frequency components in the system. The next step is to calculate the arrival time of the wave from the faultpoint to the source and to do this it is essential to determine the time, where the current value is maximum. As the wave is distorted it becomes difficult to identify the time at which current magnitude is maximum. The smoothing and differentiation of the waveform enables us to estimate the time at which the current magnitude is highwhich in turn helps in calculating the fault distance value accurately(Schweitzer, 2014). According to double ended travelling wave method the velocity of the travelling wave and the time of arrival of the wave from fault point to source have to be known accurately tomeasure the fault distance in cables or in overhead lines. The velocity of the travelling wave depends on the inductance and capacitance,whose values are known while designing the cable (Potivejkul ,2000). It also depends on parameters like type ofinsulation material used and the medium in which the cable is placed as well as the cable physical parameters. So, whenever a fault occurs the velocity can be predetermined and it is the task to measure the time of arrival of the wave. The accurate measurement of the arrival time leads to accurate measurement of fault distance.

\section{Modelling Of the Cable}

Unlike the overhead lines, modelling of power cables is difficult because many parameters are to be considered while modelling cable which makes its design complex and costlyas shown in Fig. 1(Valle ,2000). The shape of the core, type of insulation, sheath material, screens and the cable laying configuration makes its design complex (Potivejkul , 2000). The capacitance and inductance of a cable are given as follows

$$
\begin{gathered}
\mathrm{C}=\frac{2 \pi \in_{0} \in_{r}}{\log \frac{d}{r}} \mathrm{~F} / \mathrm{m} \\
\mathrm{L}=\frac{\mu_{0}}{2 \pi} \log \frac{d}{r} \mathrm{H} / \mathrm{m}(2)
\end{gathered}
$$

Here $\epsilon_{0}$ is the permittivity of the free space and its value is approximately taken as $8.854 * 10^{-12} \mathrm{~F} / \mathrm{m}$. $\in_{r}$ is the relative permittivity and its value varies depending on the insulation material used. $\mu_{0}$ is the permeability of the free space and its value is taken as $4 \pi^{*} 10^{-7} \mathrm{~N} / A^{2}$. Here $\mathrm{d}$ is the geometric mean distance and the $\mathrm{r}$ is the radius of the cable.

The capacitance in each core of a three core cable is taken as $\mathrm{C}=\mathrm{Cs}+3 \mathrm{Cc}$ where $\mathrm{Cs}$ is the capacitance of the core to sheath and $\mathrm{Cc}$ is the core to core capacitance (Potivejkul, 2000). The capacitance effect is more in underground cables compared to overhead lines because of the small separation between the conductors. Therefore the high charging current flows in the cable and also the Ferranti effect is high (Wu, 2009). Due to these two limitations the cable length is taken small. In this paper a cable of $30 \mathrm{Km}$ length is considered while the fault is created at $7 \mathrm{Km}$ from the source ' 1 '.

The surge impedance can be calculated from the known $\mathrm{L}$ and $\mathrm{C}$ values which help in knowing the surge impedance loading power of the system and over voltages caused by switching or lightning transients. Due to the high capacitance effect in cables the surge impedance value of cable is less compared to overhead lines and it depends on the operating voltage of the system(Potivejkul, 2000). For a $220 \mathrm{kV}$ underground cable system the calculated surge impedance is about $40 \mathrm{ohms}$ approximately(Wu, 2009).

$$
Z_{c}=\sqrt{\frac{L}{c}}(3)
$$




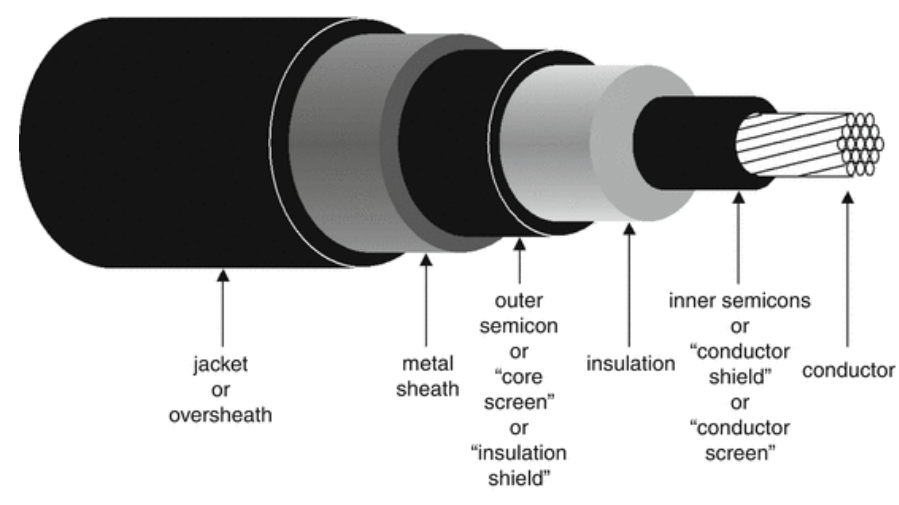

Figure 1. Construction of the underground cable

The travelling wave velocity in the power cable depends on the insulation medium which has been taken in the cable. In this paper cross linked polyethylene is considered as the insulation medium whose wave velocity is taken as $\mathrm{V}=1.72 * 10^{8} \mathrm{~m} / \mathrm{s}$. Velocity of a wave in medium is less compared to free space because velocity is dependent on permeability $\left(\mu_{r}\right)$ and permittivity $\left(\epsilon_{r}\right)$ of the medium[8]. In cables $\mu_{r}$ is taken as 1 since non magnetic material is taken and $\epsilon_{r}$ value is more in medium compared to air. Here permittivity of the medium is taken as 3.0422. ' $\mathrm{v}$ ' is the speed of the light in $\mathrm{m} / \mathrm{s}$ (Potivejkul, 2000).

$$
\begin{gathered}
\mathrm{V}=\frac{v}{\sqrt{\mu_{r} \epsilon_{r}}} \mathrm{~m} / \mathrm{s} \\
\mathrm{V}=\frac{1}{\sqrt{L C}} \mathrm{~m} / \mathrm{s}
\end{gathered}
$$

\section{Approach To Locate Fault In The Underground Cables}

\subsection{Principle Of The Travelling Wave Based Approach}

Whenever a fault occurs in a cable at some distance the voltage and current surges are produced from the fault point and they travel towardsthe either ends of the cable. The change in the voltage and current values at the fault point creates the discontinuation in the cable parameters and it results in producing the travelling waves[9].

The resistance and reactance of the cable results in damping of the travelling wave surges in the cable. The travelling waves in underground cables travels with a velocity less than that in overhead transmission lines because of the presence of relative dielectric coefficient whose value is greater than 1 in the underground cables (Chen, 2011). The velocity of a travelling wave depends on the frequency of the waveform. In an ideal step like waveform the travelling wave propagates with different velocities causing the initial step of the wave to get distorted. Thus it causes steepness in the travelling wave rising edge which has an impact on the value of the travelling wave arrival time.

\subsection{Phenomena of the double ended travelling wave method}

In the double ended travelling wave based approach two sources are placed at either ends of the line. Whenever a fault occurs at any point in the cable the wave gets reflected and refracted from the fault location (Gilany , 2007). In this method the arrival times of the first reflected and refracted waves are considered to calculate the fault distance because of which the complexity of separating the reflected waves as done in single end method decreases (Spoor , 2006). In the proposed work the smoothing and differentiation is done to reduce the steepness in the travelling wave rising edge by which dispersion effect is decreased and the accurate arrival time is determined. The fault distance calculated in the travelling wave methods depends on the wave velocity as seen in (6) where the accurate fault distance can be known when the exact value of the velocity of the travelling wave is known. A communication channel is placed in between the two ends, so that fault distance can be found approximately from the known arrival times and the wave velocity (Guzmán,2018; Hamidi, 2015). 


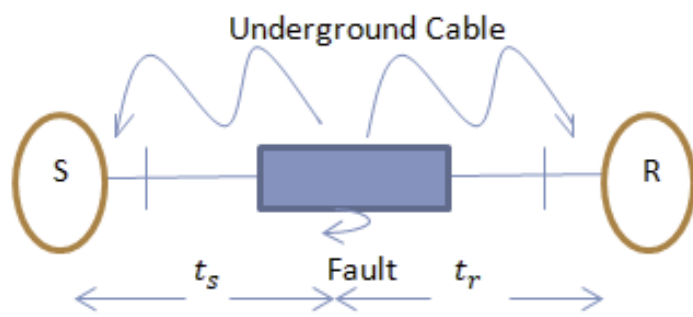

Figure 2. Propagation of travelling waves in the double end method

Where $\mathrm{L}=$ length of the cable

$$
F_{D}=\frac{1}{2}\left[L+\left(t_{s}-t_{r}\right) * V\right]
$$

$F_{D}=$ Fault distance

$t_{s}=$ Time taken to travel from fault point to ' $\mathrm{S}$ '

$t_{r}=$ Time taken to travel from fault point to ' $\mathrm{R}$ '

$\mathrm{V}=$ Velocity of the travelling wave

\subsection{Phase to Modal Transformation}

The phase or symmetrical components $(\mathrm{A}, \mathrm{B}, \mathrm{C})$ are commonly used for analysis in the power system. In this paper to locate a fault we rely on the modal components alpha, beta and zero $(\boldsymbol{\alpha}, \boldsymbol{\beta}, \mathbf{0})$ to simplify the analysis. The relationship can be developed between symmetrical and modal components using Clarke's transformation (Schweitzer ,2014). The modal components differ based on the propagation velocity, dispersion and attenuation effect and these factors affect the fault distance value (Hamidi, 2015).

$$
\left[\begin{array}{c}
I_{o} \\
I_{\alpha} \\
I_{\beta}
\end{array}\right]=T_{C}^{-1}\left[\begin{array}{c}
I_{A} \\
I_{B} \\
I_{C}
\end{array}\right]=\frac{1}{3}\left[\begin{array}{ccc}
1 & 1 & 1 \\
2 & -1 & -1 \\
0 & \sqrt{3} & -\sqrt{3}
\end{array}\right]\left[\begin{array}{c}
I_{A} \\
I_{B} \\
I_{C}
\end{array}\right]
$$

The clarke's components calculated with respect to phase A works for Phase A to ground fault and the line to line fault i.e.(BC fault). So the three sets of clarke's components are generally considered in order to analyse all types of faults.

$$
\begin{aligned}
& {\left[\begin{array}{l}
I_{\alpha}{ }^{A} \\
I_{\beta}{ }^{A} \\
I_{0}{ }^{A}
\end{array}\right]=\frac{1}{3}\left[\begin{array}{ccc}
2 & -1 & -1 \\
0 & \sqrt{3} & -\sqrt{3} \\
1 & 1 & 1
\end{array}\right]\left[\begin{array}{l}
I_{A} \\
I_{B} \\
I_{C}
\end{array}\right]} \\
& {\left[\begin{array}{l}
I_{\alpha}{ }^{B} \\
I_{\beta}{ }^{B} \\
I_{0}{ }^{B}
\end{array}\right]=\frac{1}{3}\left[\begin{array}{ccc}
-1 & 2 & -1 \\
-\sqrt{3} & 0 & \sqrt{3} \\
1 & 1 & 1
\end{array}\right]\left[\begin{array}{l}
I_{A} \\
I_{B} \\
I_{C}
\end{array}\right]} \\
& {\left[\begin{array}{l}
I_{\alpha}{ }^{C} \\
I_{\beta}{ }^{C} \\
I_{0}{ }^{C}
\end{array}\right]=\frac{1}{3}\left[\begin{array}{ccc}
-1 & -1 & 2 \\
\sqrt{3} & -\sqrt{3} & 0 \\
1 & 1 & 1
\end{array}\right]\left[\begin{array}{l}
I_{A} \\
I_{B} \\
I_{C}
\end{array}\right]}
\end{aligned}
$$

The Zero sequence components exist if equal rating of current flows through the phase A, B, C and backs to the earth. So it has higher dispersion and attenuation effect than the other two modes of components (Schweitzer ,2014). So we consider only $\boldsymbol{\alpha}$ and $\boldsymbol{\beta}$ components to locate the fault point. The alpha current exists for all types of faults whereas beta currents are preferably considered for the phase to phase faults as they provide high magnitude currents for this type of faults.

\subsection{Methodology to locate the fault distance in the cable}

The current components $I_{\alpha}, I_{\beta}$ and $I_{o}$ obtained after clarke's transformation are distorted. Hence it becomes difficult to determine the time at which the current magnitude is maximum. In turn it becomes complex to identify the arrival time of a travelling wave. To avoid this complexity initially the zero sequence current components are 
eliminated as they have higher attenuation and dispersion effect compared to alpha and beta components. Then after filtering of alpha and beta current components is done to eliminate the power frequency $(50 \mathrm{~Hz}$ or $60 \mathrm{~Hz})$ components and the low frequency components in the system. The High pass filter is used to remove the low frequency current components.

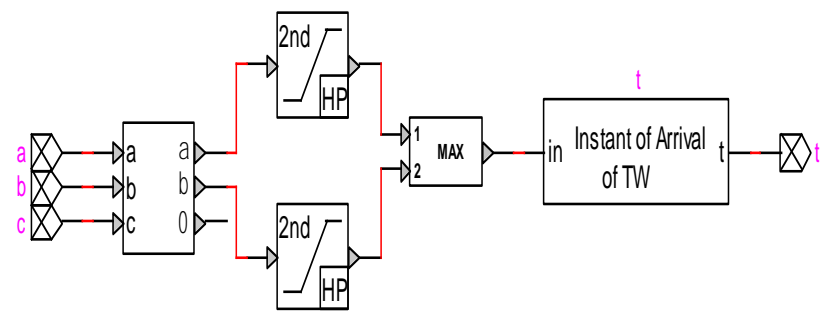

Figure 3. Sub circuit model to determine the travelling wave arrival time

After filtering, one of the alpha and beta current components is available and there are multiple maximum points present in the current waveforms which are overlapped due to the reflections and refractions in the wave. This limits the application of time sampling the current waveforms. In this approach finite rate of sampling causes large error of quantization. However filtering then after curve fitting and finally interpolation may overcome this issue but this approach cannot determine the time at which current value is high in the waveform and this drawback limits its application. Another approach is taken here to overcome this drawback in which the current waveform is first smoothed to eliminate the distortions in the current waveform and it smooth's the rising edge of the travelling wave by which it becomes less steep. This reduces the dispersion effect in the proposed work and arrival time can be determined accurately. The smoothed wave form is then differentiated to turn in to a soft pulse like shape from a step like waveform. The peak value exists at the time of the steepest slope of the current waveform. This method provides an accuracy of about 0.2 micro seconds.

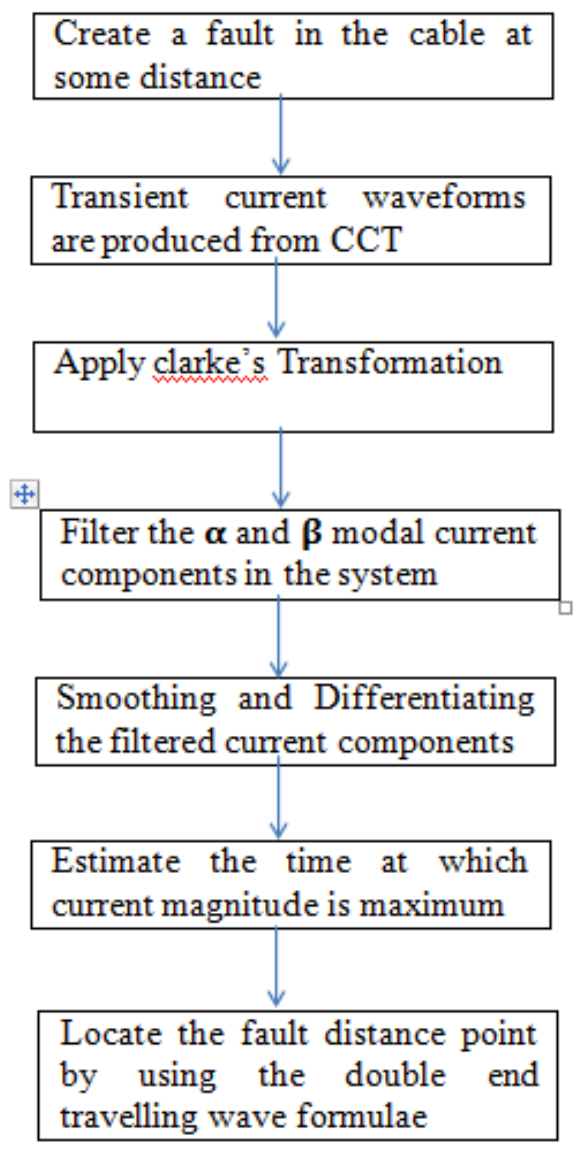

Step by step process of proposed methodology to locate a fault in the underground cable 


\section{Emtp Model and Simulations}

In this circuit model a $220 \mathrm{kVRMS}$ voltage sources are taken and placed at both ends of the cable. A current transformer is taken so as to get the symmetrical components of 3 phase fault current(A,B and C) waveforms and then by using the clarke's transformation these three phase currents are converted in to two phase $(\boldsymbol{\alpha}$ and $\boldsymbol{\beta})$ orthogonal vector current components. The fault current value is about 5 to 10 times the rated current value.The high frequency components of these two orthogonal current vectors are extracted using the high pass filter. The obtained current waveform is smoothen so as to reduce the distortions and the waveform to become less steep which results the rising edge to become smooth. The differentiation helps in converting the waveform in a step shape to a soft pulse like shape. It has its peak at the steepest slope of the current waveform. The output waveform looks in a parabola shapes owe use a parabola type interpolation method to calculate the fault distance. The fault distance depends on various parameters like ground resistance, fault inception angle, different fault types etc... and it varies accordingly (Qin,2007).

In the proposed work a cable of $30 \mathrm{Km}$ length is taken and a fault is created at $7 \mathrm{Km}$ length from the source " 1 " which means that the fault is at $23 \mathrm{Km}$ length from the source " 2 ". The surge impedance of the cable is taken as $40 \Omega$ and the wave velocity is taken as $1.72 * 10^{8} \mathrm{~m} / \mathrm{s}$ which is calculated value for cross linked polyethylene insulation medium. The calculated resistance value of the cable for a XLPE insulation medium is $0.027 \mathrm{ohms}$ (Potivejkul,2000).

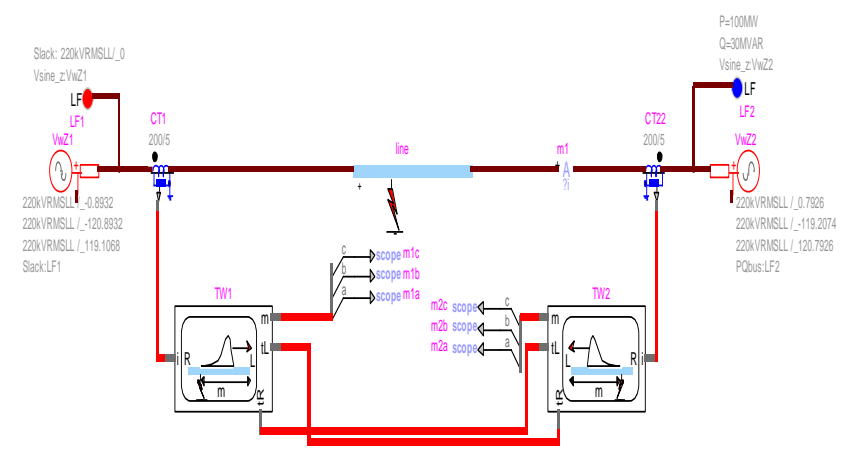

Figure 4. EMTP model to locate a fault in the underground cable

EMTP is the most advanced software used for the simulation of the electromagnetic and electromechanical transients in the time domain. It gives the most accurate and the fastest results. The circuit model created in the EMTP-RV software as shown in Fig. 4 is made to run by creating various faults and the fault distance is measured accordingly. Then after the effect of the change in ground resistance, fault inception angle and the different faults on the fault distance is analysed. It is observed that without fault the current waveform is smooth without any distortions and the transients. Then after it is observed that after the fault is created in the system the current waveforms are distorted and transients are developed in the waveforms. These transients can be analysed quickly and accurately in the time domain using the proposed travelling wave method. The time at which the fault is created is taken as 0.1 seconds and the total time of the simulation is taken as 0.3 seconds. The integration time step is taken as 0.02 seconds. It is to be noted that the integration time step should be always less than the propagation time.

\section{Results}

The fault current waveforms produced after creating LG,LL and LLL faults in the designed circuit model to identify the fault distance are as shown in Fig. 5,Fig. 6 and Fig. 7 respectively. Initially cable is made to run under smooth condition that is without creating a fault and is observed that the amplitude of the current is about 550 amps. Then after a fault is created at 0.1 seconds in phase A with respect to ground that is LG fault is created. 


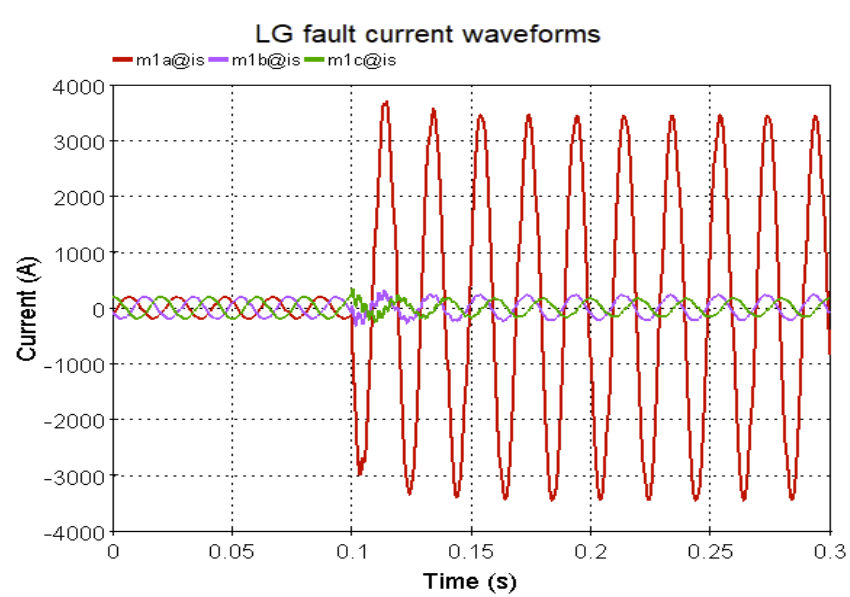

Figure 5. LG Fault current waveforms

The faulted transient current waveform for a LG fault is shown in Fig. 5. It is seen that in phase A transients are developed suddenly at 0.1 seconds and it continues. These are the symmetrical fault current waveforms and the magnitude of the fault current is 3297 amps which is about 5-6 times the rated current value.

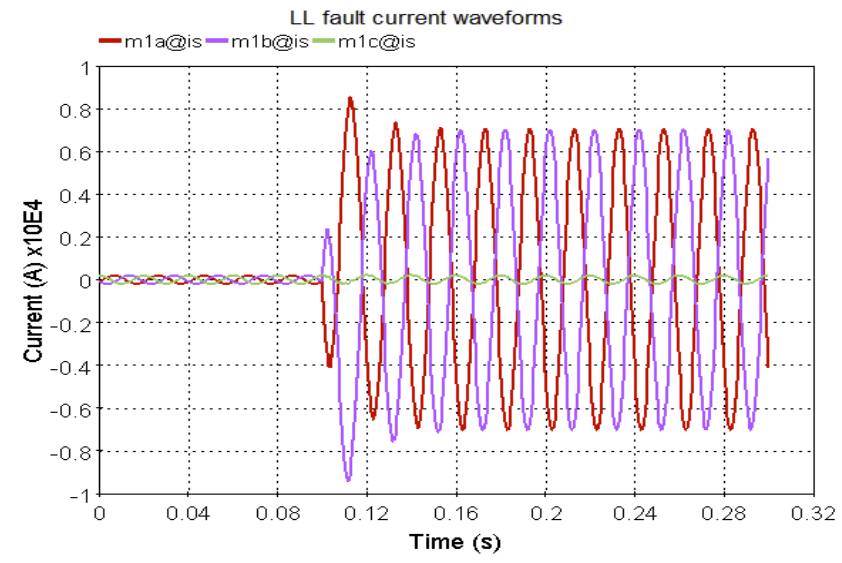

Figure 6. LL Fault current waveforms

The transient fault current waveforms for a LL fault are shown in Fig. 6. Here a fault is created in Phase A and Phase $\mathrm{B}$ at 0.1 seconds and Phase $\mathrm{C}$ is left undisturbed. The average magnitude of a fault current waveform is observed to be 6914 amps which is about 12 times the rated current value.

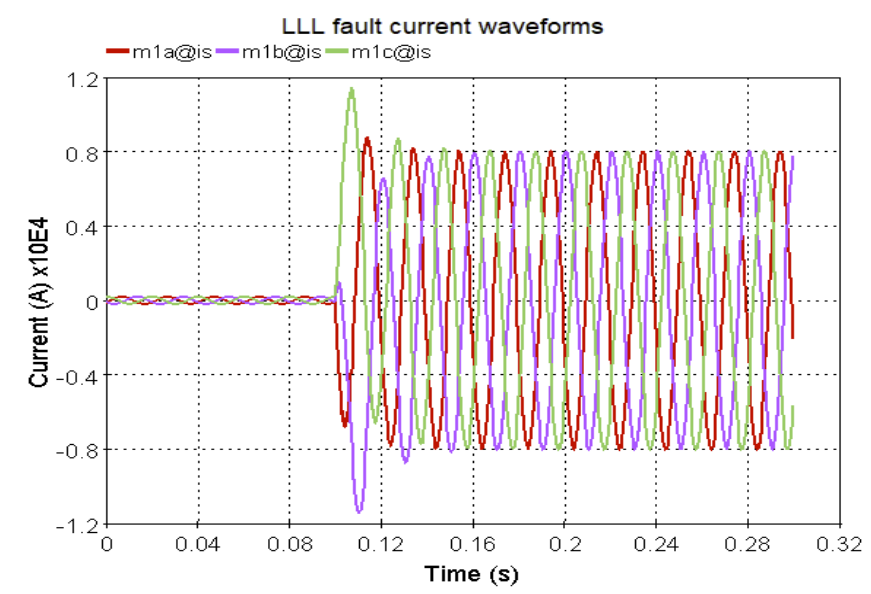

Figure 7. LLL Fault current waveforms 
The LLL fault is created in the cable by short circuiting the Phase A,B and C at 0.1 seconds. The average amplitude of the fault current waveform is about 8500 amps which is approximately 15 times the rated current value.

From the Fig. 5, Fig. 6 and Fig. 7 it can be concluded that the fault current magnitude for a LLL fault is more compared to LL and LG whereas the magnitude of the LL fault is more compared to the LG fault.

The main aim of the project is to calculate the fault distance value accurately in the power cable. To do this a communication channel is designed in the EMTP software which identifies the time taken by these transient current waveforms to reflect and refract to the sources ' 1 ' and ' 2 'which are called as the arrival times. The calculated arrival times enables us to identify the fault distance from either ends of the cable[14].

The output of the circuit model which shows the calculated fault distance value in the cable can be seen in Fig. 8 and Fig. 9 where the fault distance is calculated from the either of the sources to the fault point in the cable. It can be seen that at 0.1 seconds there is a sudden variation in the waveform which is plotted between time on $\mathrm{X}$ axis and distance on $\mathrm{Y}$ axis.

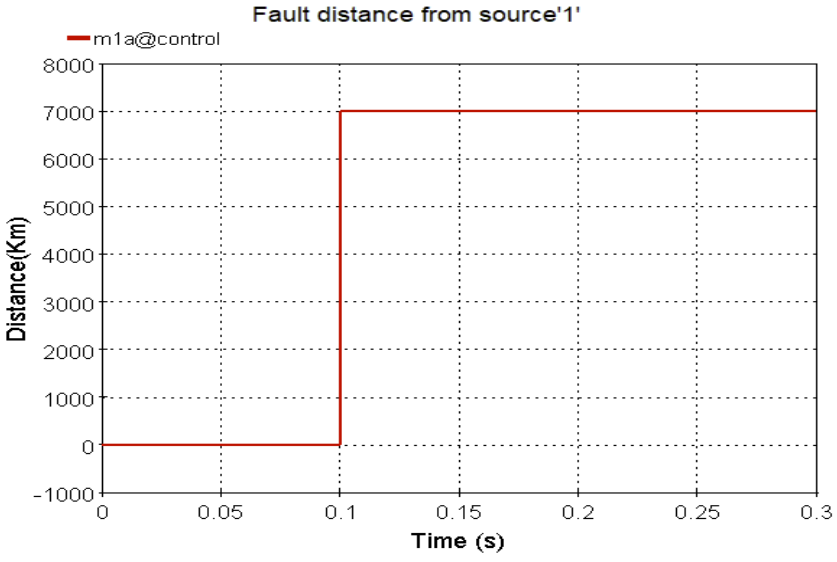

Figure 8. Fault distance from source ' 1 '

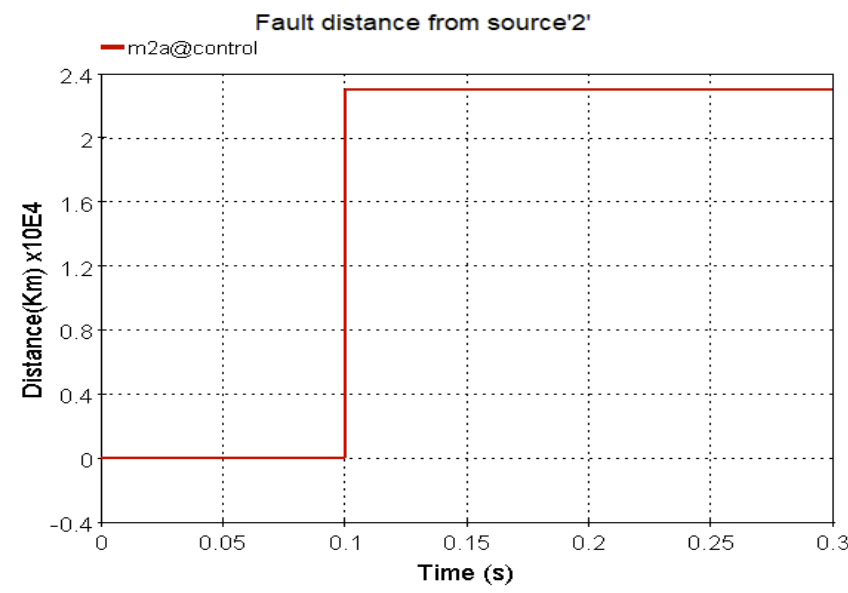

Figure 9. Fault point distance from source ' 2 '

From Fig. 8 we can say that there is a fault at $7 \mathrm{~km}$ from the source ' 1 ' and from Fig. 9 it is seen that there is a fault at $23 \mathrm{Km}$ from the source ' 2 '. By adding fault distances from the either ends of the cable we get the total length of the cable that is $30 \mathrm{Km}$. Thus we can say that the results provided by the double ended travelling wave method using the developed circuit model in the EMTP-RV are accurate.

The Simulink model developed in the EMTP software can be used for any type of cable without any practically implemented length limitations. The average accuracy of the result provided is about $93 \%$ to $100 \%$. To verify the accuracy and the limitations of the present model it has been made to run under various ground resistance values, fault types and the fault inception angles[14]. The results provided for this are tabulated in Table I, Table II and Table III respectively. 
Table 1. Fault distance calculated for various ground resistance values

\begin{tabular}{|c|c|c|c|c|c|}
\hline S.No & Fault type & $\begin{array}{c}\text { Fault distance } \\
\text { from source ' } \mathbf{~} \\
(\mathbf{L 1} \text { in Km) }\end{array}$ & $\begin{array}{l}\text { Error } \\
(\mathbf{\%})\end{array}$ & $\begin{array}{c}\text { Fault distance } \\
\text { from source '2 } \\
(\mathbf{L 2} \text { in Km) }\end{array}$ & Error (\%) \\
\hline 1 & LG & 7.00 & 0 & 23.00 & 0 \\
\hline 2 & LL & 6.977 & 0.32 & 23.023 & 0.1 \\
\hline 3 & LLL & 6.975 & 0.35 & 23.025 & 0.108 \\
\hline
\end{tabular}

The impact of the various ground resistance values on the model which is developed is as shown in Table I. The change in the ground resistance value varies the voltage and current components along the cable. The currents extracted using the CTs in the proposed work vary accordingly to the

Table 2. Fault distance calculated for different types of faults

\begin{tabular}{|c|c|c|c|c|c|}
\hline S. No & $\begin{array}{l}\text { Ground } \\
\text { Resistance } \\
(\boldsymbol{\Omega})\end{array}$ & $\begin{array}{l}\text { Fault distance } \\
\text { from source } \\
\text { '1' }(\mathrm{L1} \text { in } \\
\mathrm{Km})\end{array}$ & Error (\%) & $\begin{array}{l}\text { Fault distance from source } \\
\text { '2' (L2 in Km) }\end{array}$ & Error (\%) \\
\hline 1 & 0 & 7 & 0 & 23 & 0 \\
\hline 2 & 100 & 6.977 & 0.32 & 23.023 & 0.1 \\
\hline 2 & 200 & 6.977 & 0.32 & 23.023 & 0.1 \\
\hline 3 & 300 & 6.976 & 0.342 & 23.024 & 0.104 \\
\hline 4 & 400 & 6.975 & 0.35 & 23.025 & 0.108 \\
\hline 5 & 500 & 6.975 & 0.35 & 23.025 & 100 \\
\hline 6 & 900 & 0 & 100 & 0 & \\
\hline
\end{tabular}

change in the ground resistance value. Therefore, it becomes difficult to find out the time at which the current magnitude is maximum and this has a great impact on calculating the arrival times of the travelling waves. Since the fault distance depends on the arrival times the change in the ground resistance value varies the fault distance in the power cable.

The effect of the various faults that is LG, LL and LLL on the developed model is analyzed. It is observed that for LG fault it provides results with $100 \%$ accuracy and for LL fault it shows percentage error which is less compared to LLL fault. From the proposed work we can calculate fault distance for any type of fault accurately and the percentage error is very less which can be neglected.

In the Table III we can see how the fault distance is varied with the change in the fault inception angle in the proposed work.

\begin{tabular}{|l|l|l|l|l|l|}
\hline S.No & $\begin{array}{l}\text { Fault } \\
\text { inception } \\
\text { angle(degrees) }\end{array}$ & $\begin{array}{l}\text { Fault distance from } \\
\text { source '1' (L1 in }\end{array}$ & $\begin{array}{c}\text { Error } \\
\text { Km) }\end{array}$ & $\begin{array}{c}\text { Faul distance } \\
\text { from source '2' }\end{array}$ & Error (\%) \\
\hline 1 & 0 & 6.998 & 0.028 & 23.002 & 0.008 \\
\hline 2 & 30 & 6.998 & 0.028 & 23.002 & 0.008 \\
\hline 2 & 60 & 6.996 & 0.057 & 23.004 & 0.01 \\
\hline 3 & 90 & 7.022 & 0.314 & 22.978 & 0.095 \\
\hline 4 & 180 & 7.025 & 0.357 & 22.975 & 0.108 \\
\hline
\end{tabular}


The change in the fault inception angle varies the transient current values in the system. This change has an impact on the arrival times of the travelling wave by which fault distance can be varied. For the various fault inceptions angles the fault distance calculated is as shown in Table III. It is observed that the model developed is not sensitive to the change in fault inception angle. For the angle of 0,30 and 60 degrees it has shown the accurate fault distance value with a negligible error. For the inception angle of 90 and 180 degrees there is a percentage error which is more compared to other but it is within the acceptable limits.

\section{Conclusion}

In the proposed work the average percentage error for the calculatedfault distance value in the underground cables has been limited to 0.22 . This method does not consider the parameters which are the sources of error while calculating the fault distance value as found in impedance based method thus it improves the accuracy. It is also proved that the proposed method is insensitive to the change in the values of ground resistance, fault inception angle and the different types of faults. This type of fault locators built in the underground cables adds less cost to the power system and it is easy to use by which a safe and reliable operation of the system is possible.

\section{References}

1. Aroulanandam VV, Latchoumi TP, Balamurugan K, Yookesh TL. (2020) Improving the Energy Efficiency in Mobile Ad-Hoc Network Using Learning-Based Routing, Revue d'Intelligence Artificielle, Vol 34(3), pp. 337-343. DOI: https://doi.org/10.18280/ria.340312

2. Balamurugan K. Metrological changes in surface profile, chip, and temperature on end milling of M2HSS die steel. International Journal of Machining and Machinability of Materials, 22(6):443-453.

3. Chen, P., Wang, K. (2011) Fault location technology for high-voltage overhead lines combined with underground power cables based on travelling wave principle," in Proc. APAP, Beijing, China, vol. 1, pp. 784-751.

4. Deepthi T, Balamurugan K, Balamurugan P. (2020) Parametric Studies of Abrasive Waterjet Machining parameters on Al/LaPO4 using Response Surface Method. InIOP Conference Series: Materials Science and Engineering 2020 Dec 1 (Vol. 988, No. 1, p. 012018). IOP Publishing.

5. Ezhilarasi TP, Dilip G, Latchoumi TP, Balamurugan K. (2020) UIP-A Smart Web Application to Manage Network Environments. InProceedings of the Third International Conference on Computational Intelligence and Informatics, pp. 97-108, Springer, Singapore.

6. Gilany, M., Ibrahim, D. K., Tag Eldin, E. S. (2007) Traveling-wave-based faultlocation scheme for multiend-aged underground cable system", IEEE Transactions on Power Delivery, vol. 22, no. 1, pp. 82-89, 2007.

7. Garikapati P, Balamurugan K, Latchoumi TP, Malkapuram R. (2020) A Cluster-Profile Comparative Study on Machining AlSi $7 / 63 \%$ of SiC Hybrid Composite Using Agglomerative Hierarchical Clustering and K-Means. Silicon. Jun 3:1-12.

8. Guzmán, A., Smelich, G., Sheffield, Z. Taylor, D. (2018) Testing Traveling-Wave Line Protection and Fault Locators," proceedings of the 14th International Conference on Developments in Power System Protection, Belfast, United Kingdom.

9. Hamidi,R. J., Livani, H. (2015) A travelling wave-based fault location method for hybrid three-terminal circuits," in Proc. IEEE Power Energy Soc. Gen.Meeting, Denver, CO, USA, pp. 1-5,.

10. Latchoumi TP, Reddy MS, Balamurugan K. (2020) Applied Machine Learning Predictive Analytics to SQL Injection Attack Detection and Prevention. European Journal of Molecular \& Clinical Medicine.;7(2); pp 3543-3553.

11. Liang Rui, SUN Shixiang (2013)A combined method for single-ended traveling wave fault location", Power System Technology, Vol.37,pp.699-709,2013.

12. Qin, J., Ge, W.,et al.,(2007) Analysis on main influencing factors for transmission lines fault location precision based on traveling wave," Power system technology,vol.31, 
no.2,pp.27-35,2007.

13. Potivejkul, S., Kerdonfag, P., Jamnian, S., Kinnares,V. (2000) Design of lowvoltage cable fault detector," in Proc. IEEE Power Engineer. Society.Winter Meeting, 1 :724729.

14. Roostaee, S. Thomas, M.S. \&Mehfuz, S. (2017) Experimental studies on impedance based fault location for long transmission lines". Prot Control Mod Power Syst 2, 16 .

15. Sneha P, Balamurugan K, Kalusuraman G. (2020) Effects of Fused Deposition Model parameters on PLA-Bz composite filament. InIOP Conference Series: Materials Science and Engineering 2020 Dec 1 (Vol. 988, No. 1, p. 012028). IOP Publishing.

16. Stringfield, T.W.,.Marihart, D.J, Stevens, R.F. (1957) Fault location methods for Overhead lines",Transactions of the American Institute of Electrical Engineers. Part III:Power Apparatus and Systems, 76 (3): .518-529.

17. Schweitzer, E. O., III, A. Guzmán, M. Mynam, V. Skendzic,B. Kasztenny, C. Gallacher, and S. Marx (2016) Accurate Single-End Fault Location and Line-Length Estimation Using Traveling Waves, " proceedings of the 13th International Conference on Developments in Power System Protection, Edinburgh, United Kingdom, March 2016

18. Spoor D., Zhu, J.G. (2006) Improved single-ended traveling -wave fault-location algorithm based on experience with conventional substation transducers," IEEE Transactions on Power Delivery, Vol.21,no.3,pp.1714-1720.

19. Schweitzer, E. O., Guzmán, A., Mynam, M. V., Skendzic, V., Kasztenny, B., Marx, S.(2014) Locating Faults by the Traveling Waves They Launch," proceedings of the 67th Annual Conference for Protective Relay Engineers, College Station.

20. YIN Xiaoguang, SONG Linlin, YOU Zhi,etal (2011) Study of fault locating for transmission line double terminal traveling waves unrelated to wave speed", Power system protection and control, 39:35-39.

21. Wu, Z., Li, Z., Qi, X. et al.,( 2009) A novel double terminal traveling wave fault location method not influenced by wave speed," in Proceedings of the IEEE Transmission and Distribution Conference and Exposition. 